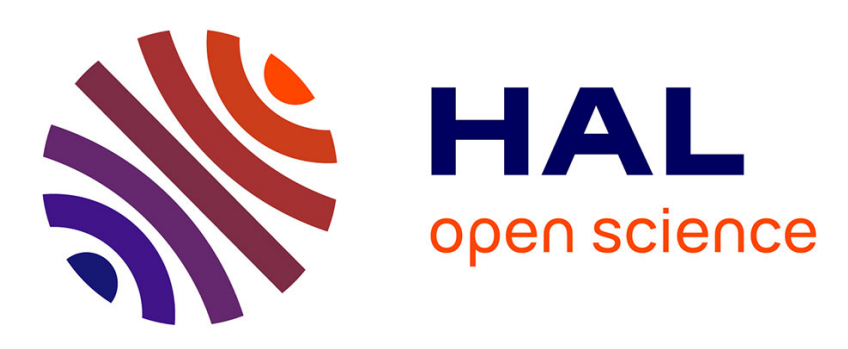

\title{
Domaine de température des phénomènes prétransitionnels dans la phase isotrope des cristaux liquides nématiques
}

\author{
J.C. Filippini, Y. Poggi
}

\section{To cite this version:}

J.C. Filippini, Y. Poggi. Domaine de température des phénomènes prétransitionnels dans la phase isotrope des cristaux liquides nématiques. Journal de Physique Lettres, 1976, 37 (1), pp.17-19. 10.1051/jphyslet:0197600370101700 . jpa-00231220

HAL Id: jpa-00231220

https://hal.science/jpa-00231220

Submitted on 1 Jan 1976

HAL is a multi-disciplinary open access archive for the deposit and dissemination of scientific research documents, whether they are published or not. The documents may come from teaching and research institutions in France or abroad, or from public or private research centers.
L'archive ouverte pluridisciplinaire HAL, est destinée au dépôt et à la diffusion de documents scientifiques de niveau recherche, publiés ou non, émanant des établissements d'enseignement et de recherche français ou étrangers, des laboratoires publics ou privés. 


\title{
DOMAINE DE TEMPÉRATURE DES PHÉNOMÈNES PRÉTRANSITIONNELS DANS LA PHASE ISOTROPE DES CRISTAUX LIQUIDES NÉMATIQUES
}

\author{
J. C. FILIPPINI et Y. POGGI \\ Laboratoire d'Electrostatique C.N.R.S., 166 X 38042 Grenoble Cedex, France
}

(Reçu le $1^{\mathrm{er}}$ octobre 1975, accepté le 31 octobre 1975)

\begin{abstract}
Résumé. - On a examiné sur un certain nombre de cristaux liquides nématiques en phase isotrope (MBBA, EBBA, MPT, HBN, PBN) le domaine de températures dans lequel ont lieu les phénomènes prétransitionnels décrits par le modèle de De Gennes. On a déterminé son étendue, environ $15^{\circ} \mathrm{C}$, à partir de mesures de biréfringence électrique, ou de biréfringence magnétique pour les substances à anisotropie négative.
\end{abstract}

Abstract. - The temperature range of pretransitional phenomena described by de Gennes's model has been examined for some nematic liquid crystals in the isotropic phase : MBBA, EBBA, MPT, HBN, PBN. Determination by electric birefringence measurements, or magnetic birefringence measurements for substances with negative anisotropy, shows it to be approximately $15^{\circ} \mathrm{C}$.

1. Introduction. - Le modèle de De Gennes [1] pour la transition entre la phase nématique et la phase isotrope d'un cristal liquide prévoit qu'en phase isotrope, près de la transition, l'intensité de la lumière diffusée, les biréfringences électrique et magnétique varient avec la température comme $\left(T-T^{*}\right)^{-\gamma}$, $T^{*}$ étant une température légèrement inférieure à la température de transition $T_{c}$, et $\gamma$ étant un exposant égal à 1 dans une théorie de champ moyen. L'expérience a vérifié ce résultat avec $\gamma=1$ pour l'intensité de la lumière diffusée [2], la biréfringence magnétique [3], la biréfringence électrique $[4,5]$, et la différence $T_{\mathrm{c}}-T^{*}$ a été déterminée aussi bien à partir de mesures de biréfringence magnétique que de mesures de biréfringence électrique pour divers cristaux liquides nématiques [6].

Lorsque la température augmente et s'écarte de $T_{\mathrm{c}}$, l'ordre à courte portée responsable du phénomène diminue et, à partir d'une certaine température, la loi en $\left(T-T^{*}\right)^{-1}$ ne doit plus être suivie : loin de $T_{\mathrm{c}}$ les variations des constantes de Kerr et de Cotton-Mouton avec la température doivent tendre vers celles des liquides ordinaires. Les études faites jusqu'ici ayant porté sur un intervalle de température trop faible pour mettre en évidence le phénomène $[2,5,6,7]$ nous avons effectué de nouvelles mesures de manière à déterminer le domaine de température $T_{1}-T_{\mathrm{c}}$ dans lequel les phénomènes prétransitionnels obéissent à la loi prévue par le modèle théorique.

La détermination du domaine de température des phénomènes prétransitionnels peut se faire à partir de mesures sur les différentes constantes physiques qui sont affectées par le phénomène. Nous nous sommes limités aux constantes de Kerr et de Cotton-Mouton. Pour un matériau donné, on pourrait s'attendre à trouver, dans les deux cas, la même différence $T_{1}-T_{\mathrm{c}}$. En fait, si cela est vrai, comme nous le verrons, pour les matériaux à anisotropie positive, il n'en est pas de même de ceux qui ont une constante de Kerr négative comme MBBA, EBBA, PAA; leur biréfringence électrique obéit à la loi en $\left(T-T^{*}\right)^{-1}$ sur un intervalle de température plus faible que la biréfringence magnétique, à cause de l'existence d'une contribution positive à l'effet Kerr qui devient prépondérante aux températures élevées $[8,9,10]$. Un tel phénomène ne se rencontre pas dans le cas de la biréfringence magnétique, ni dans le cas de la biréfringence électrique pour les corps à constante de Kerr positive.

2. Méthodes expérimentales. - Les constantes de Kerr et de Cotton-Mouton diminuant rapidement quand la température augmente, il a été nécessaire d'adapter les méthodes expérimentales à la mesure d'effets relativement faibles.

Pour l'effet Kerr, la méthode de mesure par créneaux de tension de faible durée $(\sim 1 \mu \mathrm{s})$ est la même que celle qui a déjà été décrite [4], mais les tensions appliquées sont plus élevées (jusqu'à $20 \mathrm{kV}$ pour un écartement d'électrodes de $2 \mathrm{~mm}$ ); malgré ces champs électriques intenses appliqués au liquide, la longueur du trajet parcouru par les ions pendant la durée du créneau est négligeable par rapport à l'écartement des électrodes, et le champ électrique reste uniforme. 
Pour l'effet Cotton-Mouton, la sensibilité de la méthode précédemment utilisée [6] étant devenue insuffisante ici, un nouvel appareillage a été mis au point : le champ magnétique est fourni par une bobine de Bitter [11]; la biréfringence est mesurée au moyen d'un dispositif analogue à celui décrit par Pollard et House [12]. La figure 1 donne un schéma de ce dispositif. Le faisceau lumineux fourni par un laser hélium-néon est polarisé avant son entrée dans la cellule et analysé à la sortie de celle-ci. Polariseur et analyseur sont croisés et leur direction de polarisation est orientée à $45^{\circ}$ de la direction du champ magnétique appliqué. La biréfringence introduite par la cellule de mesure lors de l'application du champ magnétique est automatiquement compensée par une cellule de

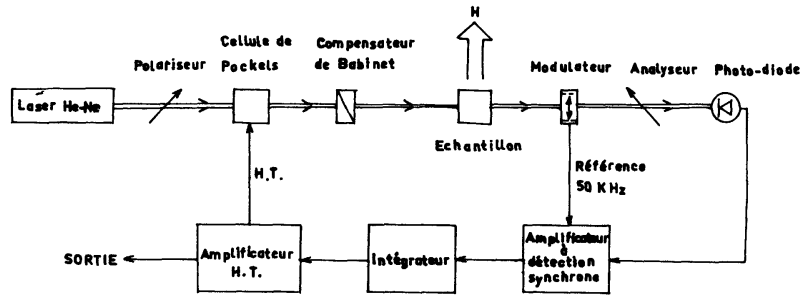

Fig. 1. - Schéma du dispositif de polarimétrie pour la mesure des constantes de Cotton-Mouton.

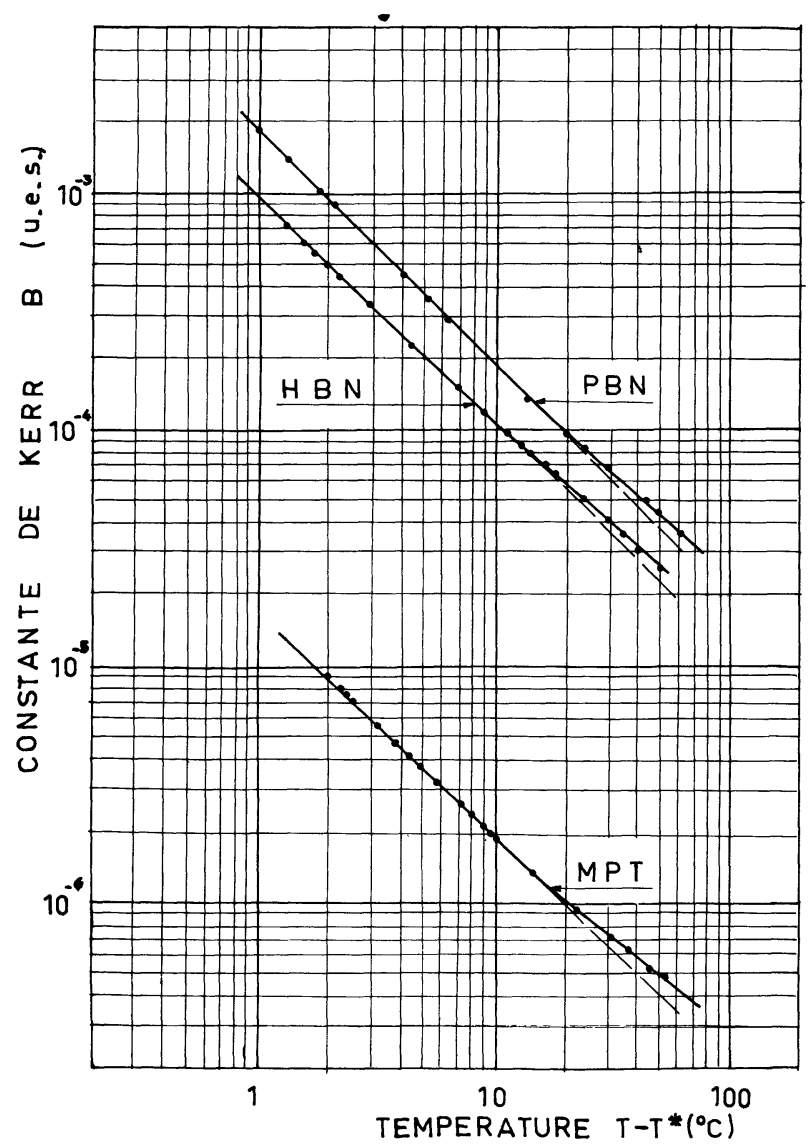

Fig. 2. - Variations de la constante de $\operatorname{Kerr} B$ de MPT, PBN et HBN en fonction de la différence de température $T-T^{*}$. Les valeurs de $T_{\mathrm{c}}$ et $T_{\mathrm{c}}-T^{*}$ sont : pour MPT, 59,1 ${ }^{\circ} \mathrm{C}$ et $1,9 \pm 0,1{ }^{\circ} \mathrm{C}$; pour $\mathrm{PBN}, 34,8^{\circ} \mathrm{C}$ et $0,7 \pm 0,2^{\circ} \mathrm{C}$; pour $\mathrm{HBN}, 41,7^{\circ} \mathrm{C}$ et $1,0 \pm 0,1^{\circ} \mathrm{C}$
Pockels dont la tension de commande est proportionnelle à cette biréfringence. Le dispositif permet de mesurer des déphasages de l'ordre de $10^{-4}$ radian. Les plus petits déphasages mis en jeu ici étant de l'ordre de $10^{-2}$ radian, la détermination des constantes de Cotton-Mouton peut se faire avec une très faible incertitude.

3. Résultats. - Les matériaux examinés furent les suivants :

- $p$-méthoxybenzylidène $p$ - $n$-butylaniline (MBBA)

- $p$-éthoxybenzylidène $p$ - $n$-butylaniline (EBBA)

- $p$ - $p^{\prime}$-méthoxypentyltolane (MPT)

- 4-cyano 4'-pentyle biphényle (PBN)

- 4-cyano 4'-heptyle biphényle (HBN).

Pour chacun d'eux, on a déterminé $T^{*}$ par extrapolation des courbes donnant l'inverse des constantes de Kerr et de Cotton-Mouton en fonction de $T$. La figure 2 donne les variations de la constante de Kerr $B$ de MPT, PBN et HBN en fonction de la différence de température $T-T^{*}$. Le domaine de température dans lequel $B$ varie comme $\left(T-T^{*}\right)^{-1}$ s'étend sur 15 à $20^{\circ}$; au-delà, la décroissance est moins rapide. Cependant, même à $80^{\circ} \mathrm{C}$ de la transition, on est encore très loin du comportement d'un liquide ordinaire. Pour MPT, de nouvelles mesures plus précises que celles faites antérieurement [4] montrent qu'après 15 à $20^{\circ} \mathrm{C}$ au-delà de $T_{\mathrm{c}}$ la décroissance est de même type que celle des autres substances exami-

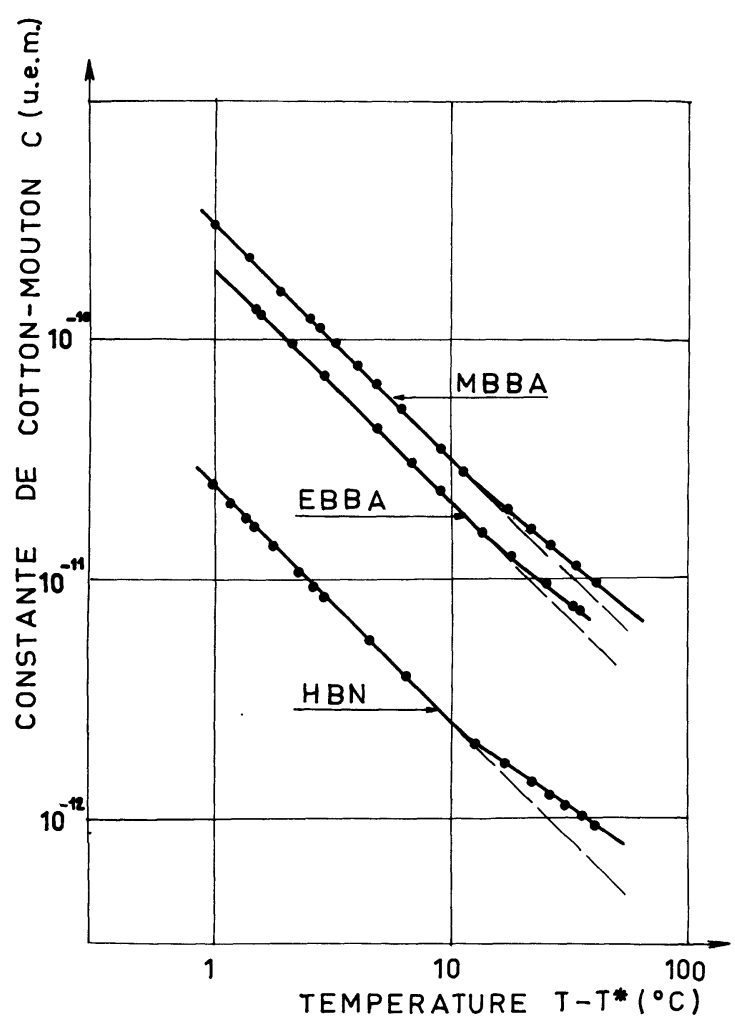

FIG. 3. - Variations de la constante de Cotton-Mouton $C$ de MBBA, EBBA et HBN en fonction de la différence de température $T-T^{*}$. Les valeurs de $T_{\mathrm{c}}$ et $T_{\mathrm{c}}-T^{*}$ sont : pour MBBA, $45,3{ }^{\circ} \mathrm{C}$ et $0,7 \pm 0,1^{\circ} \mathrm{C}$; pour EBBA, $77,8^{\circ} \mathrm{C}$ et $0,8 \pm 0,1^{\circ} \mathrm{C}$; pour HBN, $41,7^{\circ} \mathrm{C}$ et $0,9 \pm 0,1^{\circ} \mathrm{C}$. 
nées. Pour MBBA et EBBA, dont la constante de Kerr est négative, le domaine de température dans lequel $B$ varie comme $\left(T-T^{*}\right)^{-1}$ s'étend sur $12^{\circ} \mathrm{C}$ et $4{ }^{\circ} \mathrm{C}$ respectivement [4] : contrairement à ce qui se passe pour les corps à constante de Kerr positive comme MPT, PBN, HBN, les phénomènes sont modifiés par l'existence d'une contribution positive à l'effet Kerr qui tend à réduire la zone en $\left(T-T^{*}\right)^{-1}$. On doit s'attendre à trouver, par des mesures de biréfringence magnétique, une zone en $\left(T-T^{*}\right)^{-1}$ plus étendue : c'est ce que montre la figure 3 qui donne les variations de la constante de CottonMouton, $C$ en fonction de $T-T^{*}$ pour MBBA, EBBA, et aussi pour HBN. La zone en $\left(T-T^{*}\right)^{-1}$ s'étend sur environ $15^{\circ} \mathrm{C}$ pour les trois substances.

De plus, il est possible d'estimer la valeur de la longueur de cohérence $\xi(T)$ de l'ordre à courte distance, à la température à partir de laquelle cesse l'influence des phénomènes prétransitionnels purs. La théorie des cristaux liquides, considérés comme un milieu continu, prévoit que la constante de CottonMouton $C$ est proportionnelle au carré de $\xi(T)$ [13]. Pour MBBA, Chu, Bak et Lin [14] ont trouvé que, près de $T_{\mathrm{c}}, \xi(T)$ était de l'ordre de $150 \AA$ en bon accord avec l'estimation de De Gennes [13]. Nos mesures des variations de la constante de CottonMouton avec la température permettent d'estimer la valeur de la longueur de cohérence pour $T_{1}=T^{*}+15^{\circ} \mathrm{C}$ à environ $50 \AA: T_{1}$ représente la température à partir de laquelle cesse l'influence des phénomènes prétransitionnels purs obéissant au modèle de De Gennes.

\section{Bibliographie}

[1] De Gennes, P. G., Mol. Cryst. Liq. Cryst. 12 (1972) 193.

[2] Litster, J. D. and Stinson, T. W., J. Appl. Phys. 41 (1970) 996.

[3] Stinson, T. W. and Litster, J. D., Phys. Rev. Lett. 25 (1970) 503.

[4] FilipPini, J. C. et Poggi, Y., J. Physique Lett. 35 (1974) L-99.

[5] Wong, G. K. L. and Shen, Y. R., Phys. Rev. A 10 (1974) 1277.

[6] Filippini, J. C. et Poggi, Y., C.R. Hebd. Séan. Acad. Sci. B 279 (1974) 605.

[7] Schadt, M. and Helfrich, W., Mol. Cryst. Liq. Cryst. 17 (1972) 335 .

[8] Tsuetrov, V. N. and Ryumtsev, E. I., Sov. Phys. Crystallogr. 13 (1968) 225.
[9] Filippini, J. C. and Poggi, Y., Phys. Lett. 49A (1974) 291

[10] Madhusudana, N. V. and Chandrasekhar, S., Pramana 1 (1973) 12.

[11] Les expériences ont été faites au Service National des Champs Intenses à Grenoble.

[12] Pollard, A. F. and House, H., Electron. Lett. 4 (1968) 166.

[13] De Gennes, P. G., The Physics of Liquid Crystals (Clarendon Press, Oxford) 1974.

[14] Chu, B., BaK, C. S. and Lin, F. L., Phys. Rev. Lett. 28 (1972) 1111. 